PROCEEDINGS OF THE

AMERICAN MATHEMATICAL SOCIETY

Volume 129, Number 9, Pages 2607-2616

S 0002-9939(01)05878-6

Article electronically published on February 15, 2001

\title{
AUTOMORPHISMS OF COXETER GROUPS OF RANK THREE
}

\author{
W. N. FRANZSEN AND R. B. HOWLETT
}

(Communicated by Stephen D. Smith)

\begin{abstract}
If $W$ is an infinite rank 3 Coxeter group, whose Coxeter diagram has no infinite bonds, then the automorphism group of $W$ is generated by the inner automorphisms and any automorphisms induced from automorphisms of the Coxeter diagram. Indeed $\operatorname{Aut}(W)$ is the semi-direct product of $\operatorname{Inn}(W)$ and the group of graph automorphisms.
\end{abstract}

\section{Preliminaries}

The main aim of this paper is to prove the following result.

Theorem 1. If $W$ is an infinite, rank 3, Coxeter group, whose Coxeter diagram has no infinite bonds, then the automorphism group of $W$ is the semi-direct product of $\operatorname{Inn}(W)$ and the group of graph automorphisms.

We recall that a Coxeter group is a group with a presentation of the form

$$
\left.W=\operatorname{gp}\left\langle\left\{r_{a} \mid a \in \Pi\right\}\right|\left(r_{a} r_{b}\right)^{m_{a b}}=1 \text { for all } a, b \in \Pi\right\rangle
$$

where $\Pi$ is some indexing set, whose cardinality is called the rank of $W$, and the parameters $m_{a b}$ satisfy the following conditions: $m_{a b}=m_{b a}$, each $m_{a b}$ lies in the set $\{m \in \mathbb{Z} \mid m \geq 1\} \cup\{\infty\}$ and $m_{a b}=1$ if and only if $a=b$. In this paper we shall usually restrict ourselves to finite values of $m_{a b}$.

If $w \in W$, then we define $l(w)$ to be the length of the shortest expression for $w$ as a product of generators $r_{a}(a \in \Pi)$.

The (Coxeter) diagram of $W$ is a graph with vertex set $\Pi$ in which an edge (or bond) labelled $m_{a b}$ joins $a, b \in \Pi$ whenever $m_{a b} \geq 3$. We say that the group is irreducible if this graph is connected.

Let $V$ be a real vector space with basis $\Pi$, and define a bilinear form $B$ on $V$ by

$$
B(a, b)=-\cos \left(\pi / m_{a b}\right)
$$

for all $a, b \in \Pi$. For each $a \in V$ such that $B(a, a)=1$ we define $\sigma_{a}: V \rightarrow V$ by $\sigma_{a} v=v-2 B(a, v) a$; it is well known (see, for example, Corollary 5.4 of [5]) that $W$ has a faithful representation on $V$ given by $r_{a} \mapsto \sigma_{a}$ for all $a \in \Pi$. We shall identify elements of $W$ with their images in this representation; thus $r_{a}=\sigma_{a}$ is the reflection in the hyperplane perpendicular to $a$. The action of $W$ on $V$ preserves the form $B$.

Received by the editors December 1, 1999 and, in revised form, January 31, 2000.

2000 Mathematics Subject Classification. Primary 20F55.

(C)2001 American Mathematical Society 
The elements of the basis $\Pi$ are called simple roots, and the reflections $r_{a}$ for $a \in \Pi$ are called simple reflections. We call $\Phi=\{w a \mid w \in W, a \in \Pi\}$ the root system of $W$.

The following lemma collects together some facts which will be useful later.

Lemma 2. Given the above representation of the Coxeter group $W$, the following are true:

(1) If $v \in \Phi$ and $v=\sum_{a \in \Pi} \lambda_{a} a$, then either $\lambda_{a} \geq 0$ for all $a \in \Pi$ or $\lambda_{a} \leq 0$ for all $a \in \Pi$. In the former case we call $v$ a positive root, in the latter case $a$ negative root, and we define $\Phi^{+}$and $\Phi^{-}$to be the set of all positive roots and the set of all negative roots respectively.

(2) If $w \in W$ is a reflection, then $w=r_{\alpha}$ for some $\alpha \in \Phi$. Furthermore, $\alpha=x a$ for some $x \in W$ and $a \in \Pi$, whence $w=x r_{a} x^{-1}$ is conjugate to a simple reflection.

(3) For all $w \in W$ define $N(w)=\left\{v \in \Phi^{+} \mid w v \in \Phi^{-}\right\}$. Then $l(w)=|N(w)|$; in particular, $N(w)$ is a finite set.

(4) $W$ is a finite group if and only if the bilinear form $B$ is positive definite.

(5) $\Phi$ is finite if and only if $W$ is finite.

Proof. Section 5.4 in [5] contains a proof of (1), while (2) and (3) appear as Proposition 1.14 and Corollary 1.7 respectively. Theorem 4.1 in [4] includes both (4) and $(5)$.

For each $I \subseteq \Pi$ we define $W_{I}=\operatorname{gp}\left\langle\left\{r_{a} \mid a \in I\right\}\right\rangle$; these subgroups are called the standard parabolic subgroups of $W$. Clearly $W_{I}$ preserves the subspace $V_{I}$ spanned by $I$; furthermore it acts on this subspace as a Coxeter group with root system $\Phi_{I}=\Phi \cap V_{I}$. A parabolic subgroup of $W$ is any subgroup of the form $w W_{I} w^{-1}$ for some $w \in W$ and $I \subseteq \Pi$.

To save space in our later calculations we shall write $\mathrm{s}(\theta)$ for $\sin \theta$ and $\mathrm{c}(\theta)$ for $\cos \theta$. We also use $\pi_{k}$ for $\pi / k$ (for any positive integer $k$ ) and $u \cdot v$ for $B(u, v)$. It is readily checked that if $I=\{a, b\}$ is a two-element subset of $\Pi$, then $\Phi_{I}$ consists of all vectors $v$ of the form

$$
\frac{\mathrm{s}\left((h-1) \pi_{m}\right)}{\mathrm{s}\left(\pi_{m}\right)} a+\frac{\mathrm{s}\left(h \pi_{m}\right)}{\mathrm{s}\left(\pi_{m}\right)} b,
$$

where $h \in \mathbb{Z}$. Observe that $v \cdot a=-\mathrm{c}\left(h \pi_{m}\right)$ and $v \cdot b=\mathrm{c}\left((h-1) \pi_{m}\right)$. Replacing $h$ by $m-h+1$ gives the equivalent formula

$$
\frac{\mathrm{s}\left(h \pi_{m}\right)}{\mathrm{s}\left(\pi_{m}\right)} a+\frac{\mathrm{s}\left((h-1) \pi_{m}\right)}{\mathrm{s}\left(\pi_{m}\right)} b,
$$

where now $v \cdot a=\mathrm{c}\left((h-1) \pi_{m}\right)$ and $v \cdot b=-\mathrm{c}\left(h \pi_{m}\right)$. The positive roots in $\Phi_{I}$ are the vectors of the form (11) or (2) with $1 \leq h \leq m$. We see that positive numbers appearing as coefficients of $a$ or $b$ in roots $v \in \Phi_{\{a, b\}}^{+}$are never less than 1. This result in fact extends to the entire root system.

Lemma 3 (Brink [1]). Suppose that $v=\sum_{a \in \Pi} \lambda_{a} a \in \Phi^{+}$. For each $a \in \Pi$, if $\lambda_{a}>0$, then $\lambda_{a} \geq 1$.

(The proof of this proceeds by induction on the length of a shortest element $w \in W$ such that $v=w b$ for some $b \in \Pi$, the point being that if $w=w^{\prime} r_{c}$ with $l\left(w^{\prime}\right)=l(w)-1$, then $v=w^{\prime}(b+\lambda c)$ for some $\lambda \geq 1$, and the inductive hypothesis applies to $w^{\prime} b$ and $w^{\prime} c$.) 
For convenience we record the following trivial fact.

Lemma 4. Let $u, v$ be unit vectors in a Euclidean plane with $u \cdot v=\mathrm{c}(\theta) \neq \pm 1$. Then the unique $x$ in the plane such that $x \cdot u=\lambda$ and $x \cdot v=\mu$ is given by

$$
x=\frac{\lambda-\mathrm{c}(\theta) \mu}{\mathrm{s}(\theta)^{2}} u+\frac{\mu-\mathrm{c}(\theta) \lambda}{\mathrm{s}(\theta)^{2}} v .
$$

\section{Finite SUbGROUPS}

Suppose that $W$ is any Coxeter group with $\Pi$ as the set of simple roots. We shall make use of the following result, which is due to Tits and appears in [2], Exercise 2d, p. 130 .

Lemma 5. If $W$ is a Coxeter group and $H \leq W$ is finite, then $H$ is contained in a finite parabolic subgroup of $W$.

One immediate consequence of Lemma 5 is that every maximal finite subgroup of a Coxeter group is parabolic.

The following can be found as Theorem 2.7.4 of 3 .

Lemma 6 (Kilmoyer). Let $I, J \subseteq \Pi$ and suppose that $d \in W$ is the minimal length element of $W_{I} d W_{J}$. Then $W_{I} \cap d W_{J} d^{-1}=W_{K}$ where $K=I \cap d J$.

Corollary 7. Let $I, J \subset \Pi$ and $t \in W$. Then for some $\alpha \in W_{I}$,

$$
W_{I} \cap t W_{J} t^{-1}=\alpha W_{K} \alpha^{-1}
$$

where $K=I \cap d J \subseteq \Pi$, with $d$ the minimal length element of $W_{I} t W_{J}$.

Proof. We can write $t=\alpha d \beta$ where $\alpha \in W_{I}$ and $\beta \in W_{J}$, and $d$ is the minimal length element in $W_{I} t W_{J}$. By Lemma 6

$$
W_{I} \cap t W_{J} t^{-1}=\alpha\left(W_{I} \cap d W_{J} d^{-1}\right) \alpha^{-1}=\alpha W_{K} \alpha^{-1} .
$$

Suppose that $I \subseteq \Pi$ is such that $\left|W_{I}\right|$ is finite and $W_{J}$ is infinite for all $J$ with $I \varsubsetneqq J \subseteq \Pi$. Then $W_{I}$ is a maximal finite standard parabolic subgroup of $W$.

Lemma 8. If $W$ is any infinite Coxeter group, then all maximal finite standard parabolic subgroups of $W$ are maximal finite subgroups of $W$.

Proof. Suppose that $W_{I}$ is a maximal finite standard parabolic subgroup but is not a maximal finite subgroup. By Lemma 5] we can find $K \subset \Pi$ and $t \in W$ such that $W_{I} \varsubsetneqq t W_{K} t^{-1}$ where $W_{K}$ is finite. Without loss we may assume that $t$ has minimal length in $t W_{K}$. Then, by Corollary [7,

$$
W_{I}=W_{I} \cap t W_{K} t^{-1}=\alpha W_{I \cap t K} \alpha^{-1},
$$

for some $\alpha \in W_{I}$. Conjugating by $\alpha^{-1}$ shows that $W_{I}=W_{I \cap t K}$ and therefore $I=I \cap t K \subset t K$, and hence $t^{-1} I \subset K$. By hypothesis $W_{I}$ is not properly contained in a finite standard parabolic subgroup; so we know that $t \neq 1$, and thus we can find a simple root $e$ such that $t^{-1} e=f$ is negative. As $t$ has minimal length in $t W_{K}$ it takes positive roots in the root system of $W_{K}$ to positive roots. But $-f$ is a positive root while $t(-f)=-e$ is negative, and so we can conclude that $f$ is not in the root system of $W_{K}$. Thus when $f=t^{-1} e$ is expressed as a linear combination of simple roots, some $g \notin K$ appears with a negative coefficient. It follows that if $h$ is any positive root in the root system of $W_{I \cup\{e\}}$ which is not in the root system of $W_{I}$, then $t^{-1} h$ involves $g$ with a negative coefficient. But $W_{I \cup\{e\}}$ is infinite, while 
$W_{I}$ is not. So $t^{-1}$ takes an infinite number of positive roots to negative roots, and hence has infinite length, which is a contradiction.

\section{Automorphisms}

Our objective is to describe the automorphism group of $W$. Clearly symmetries of the Coxeter diagram give rise to automorphisms which permute the simple reflections; we call these graph automorphisms. They clearly form a group. We begin by showing that graph automorphisms are always outer, given only that $W$ is infinite and irreducible. The remainder of the paper is devoted to showing that these give the full outer automorphism group if $W$ is a rank three infinite Coxeter group.

Lemma 9. If $W$ is any infinite irreducible Coxeter group, then the only graph automorphism which is inner is the identity.

Proof. Suppose that conjugation by $w \in W$ is a graph automorphism. We now let $J=\left\{\alpha \in \Pi \mid w \alpha \in \Phi^{-}\right\}$. If $J=\emptyset$, then $N(w)=\emptyset$, whence $l(w)=0$ by Lemma 2] giving $w=1$. If $J=\Pi$, then $w \Phi^{+}=\Phi^{-}$and $l(w)=\left|\Phi^{+}\right|$. But this is impossible since $\Phi$ is infinite (by Lemma 2). Thus $\emptyset \varsubsetneqq J \varsubsetneqq \Pi$; so both $J$ and $\Pi \backslash J$ are non-empty.

As conjugation by $w$ is a graph automorphism, for each $\alpha \in \Pi$ there is an $\alpha^{\prime} \in \Pi$ such that $w r_{\alpha} w^{-1}=r_{\alpha^{\prime}}$. This gives $w \alpha= \pm \alpha^{\prime}$. It follows that $w J=-K$ for some $K \subset \Pi$ and $w(\Pi \backslash J)=\Pi \backslash K$. Now let $\alpha \in J$ and $\beta \in \Pi \backslash J$. Then $-w \alpha=\alpha^{\prime} \in K$ and $w \beta=\beta^{\prime} \in \Pi \backslash K$, and by the definition of $B$ it follows that $B(\alpha, \beta) \leq 0$ and $B\left(\alpha^{\prime}, \beta^{\prime}\right) \leq 0$. But $B(\alpha, \beta)=B(w \alpha, w \beta)=-B\left(\alpha^{\prime}, \beta^{\prime}\right)$, and so we conclude that $B(\alpha, \beta)=0$. Since this holds for all $\alpha \in J$ and $\beta \in \Pi \backslash J$, and the two sets are non-empty, this contradicts the irreducibility of $W$.

For the remainder let $W$ be an infinite Coxeter group, with the following diagram:

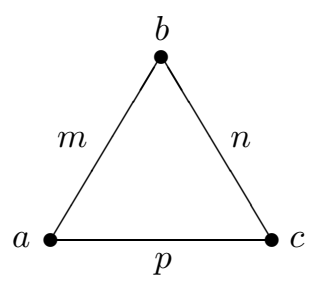

where $2 \leq m, n, p<\infty$. The group associated with this diagram is finite if $(m, n, p)$ is $(m, 2,2),(3,3,2),(4,3,2)$ or $(5,3,2)$ (permuting $m, n$ and $p$ if necessary); so these cases do not arise. Initially we shall ignore the cases $(m, n, p)=(6,3,2),(4,4,2)$ or $(3,3,3)$, where the form $B$ is degenerate. It is readily verified that the discriminant of $B$ is negative in all other cases. When $m n p$ is even we choose the labelling so that $m$ is even and $n \geq p$, while if $m n p$ is odd we choose it so that $m \geq n \geq p$. Then label the simple roots $a, b$ and $c$ as indicated in the diagram.

In view of Lemma 8 we have the following fact.

Lemma 10. The maximal finite subgroups of $W$ have the form $t W_{I} t^{-1}$ where $I=$ $\{a, b\},\{a, c\}$ or $\{b, c\}$ and $t \in W$.

Suppose $\phi$ is an automorphism of the rank three infinite Coxeter group $W$. The parabolic subgroup $\left\langle r_{a}, r_{b}\right\rangle$ is a maximal finite subgroup of $W$, and therefore $\phi\left\langle r_{a}, r_{b}\right\rangle$ is also a maximal finite subgroup; hence it follows that $\phi\left\langle r_{a}, r_{b}\right\rangle$ is 
conjugate to a standard parabolic subgroup. So, modifying $\phi$ by an inner automorphism if necessary, we may assume that $\phi\left\langle r_{a}, r_{b}\right\rangle=\left\langle r_{x}, r_{y}\right\rangle$ for some $x, y \in \Pi$. Furthermore, $\phi\left\langle r_{a}, r_{c}\right\rangle=t\left\langle r_{\alpha}, r_{\beta}\right\rangle t^{-1}$ for some $\alpha, \beta \in \Pi$ and $t \in W$, and since $\left\langle r_{a}\right\rangle=\left\langle r_{a}, r_{b}\right\rangle \cap\left\langle r_{a}, r_{c}\right\rangle$, it follows that $\phi\left\langle r_{a}\right\rangle=\left\langle r_{x}, r_{y}\right\rangle \cap t\left\langle r_{\alpha}, r_{\beta}\right\rangle t^{-1}$ is a parabolic subgroup of $W$ of order 2. Thus $\phi\left(r_{a}\right)$ is a reflection. The same holds for $r_{b}$ and $r_{c}$. This, together with Lemma 2 part 2, proves the following lemma.

Lemma 11. All automorphisms of $W$ map reflections to reflections.

Without loss of generality we may assume that $\phi\left\langle r_{a}, r_{b}\right\rangle=\left\langle r_{x}, r_{y}\right\rangle$, where $x, y \in \Pi$. If $\{x, y\}=\{a, c\}$, then $m=p$ and we have a graph automorphism fixing $r_{a}$ and interchanging $r_{b}$ and $r_{c}$; so, up to inner and graph automorphisms, $\phi$ fixes $\left\langle r_{a}, r_{b}\right\rangle$. If $\{x, y\}=\{b, c\}$ we can use a similar argument. Thus we may assume that $\phi\left\langle r_{a}, r_{b}\right\rangle=\left\langle r_{a}, r_{b}\right\rangle$.

Now $\phi\left(r_{a}\right)$ is a reflection in $\left\langle r_{a}, r_{b}\right\rangle$; so $\phi\left(r_{a}\right)=t r_{a} t^{-1}$ or $t r_{b} t^{-1}$ for some $t \in\left\langle r_{a}, r_{b}\right\rangle$. As conjugating by $t^{-1}$ fixes $\left\langle r_{a}, r_{b}\right\rangle$, we may assume that, up to inner and graph automorphisms,

$$
\begin{aligned}
\phi\left\langle r_{a}, r_{b}\right\rangle & =\left\langle r_{a}, r_{b}\right\rangle, \\
\phi\left(r_{a}\right) & =r_{a} \text { or } r_{b} .
\end{aligned}
$$

If $m$ is odd, then $r_{a}$ and $r_{b}$ are conjugate in $\left\langle r_{a}, r_{b}\right\rangle$; moreover, if $n=p$, then we have a graph automorphism that interchanges $r_{a}$ and $r_{b}$. So we only need consider the possibility that $\phi\left(r_{a}\right)=r_{b}$ when $m$ is even and $n>p$, remembering that we have labelled the graph so that $n \geq p$.

Since $\phi\left\langle r_{a}, r_{b}\right\rangle=\left\langle r_{a}, r_{b}\right\rangle$, we know that $\phi\left(r_{b}\right)$ is the reflection corresponding to some root in $\Phi_{\{a, b\}}$. We can choose this root to be positive, since a root and its negative correspond to the same reflection. Similarly, $\phi\left(r_{c}\right)$ is the reflection corresponding to some positive root. Thus the two cases we must consider are as follows:

(A) $\phi\left(r_{a}\right)=r_{a}, \phi\left(r_{b}\right)=r_{b^{\prime}}$ and $\phi\left(r_{c}\right)=r_{x}$, where $x \in \Phi^{+}$, and $b^{\prime} \in \Phi_{\{a, b\}}^{+}$has the form (1) for some $h$ such that $1 \leq h \leq m-1$ (since $\left.b^{\prime} \neq a\right)$;

(B) $\phi\left(r_{a}\right)=r_{b}, \phi\left(r_{b}\right)=r_{a^{\prime}}$ and $\phi\left(r_{c}\right)=r_{x^{\prime}}$, where $x^{\prime} \in \Phi^{+}$, and $a^{\prime} \in \Phi_{\{a, b\}}^{+}$has the form (2) for some $h$ such that $1 \leq h \leq m-1$.

In Case (A), since $r_{a} r_{x}$ and $r_{b^{\prime}} r_{x}$ must have the same orders as $r_{a} r_{c}$ and $r_{b} r_{c}$ respectively, we must have $a \cdot x=-\mathrm{c}\left(i \pi_{p}\right)$ and $b^{\prime} \cdot x=-\mathrm{c}\left(j \pi_{n}\right)$ for some integers $i$ and $j$, while in Case (B) we similarly obtain $b \cdot x^{\prime}=-\mathrm{c}\left(i \pi_{p}\right)$ and $a^{\prime} \cdot x^{\prime}=-\mathrm{c}\left(j \pi_{n}\right)$. We may choose $i$ and $j$ so that $1 \leq i \leq p-1$ and $1 \leq j \leq n-1$. We also have $a \cdot b^{\prime}=-\mathrm{c}\left(h \pi_{m}\right)$ (Case $\left.(\mathrm{A})\right)$ and $b \cdot a^{\prime}=-\mathrm{c}\left(h \pi_{m}\right)$ (Case $\left.(\mathrm{B})\right)$.

Noting that the restriction of $B$ to the subspace $V_{\{a, b\}}$ is positive definite, let proj $: V \rightarrow V_{\{a, b\}}$ be the orthogonal projection, and define $x_{\perp}=c-\operatorname{proj}(c)$, $x_{0}=\operatorname{proj}(x)$ and $x_{0}^{\prime}=\operatorname{proj}\left(x^{\prime}\right)$. By Lemma 4

$$
\operatorname{proj}(c)=-\frac{\mathrm{c}\left(\pi_{m}\right) \mathrm{c}\left(\pi_{n}\right)+\mathrm{c}\left(\pi_{p}\right)}{\mathrm{s}\left(\pi_{m}\right)^{2}} a-\frac{\mathrm{c}\left(\pi_{m}\right) \mathrm{c}\left(\pi_{p}\right)+\mathrm{c}\left(\pi_{n}\right)}{\mathrm{s}\left(\pi_{m}\right)^{2}} b
$$

and so

$$
\begin{aligned}
x_{\perp} & =\frac{\mathrm{c}\left(\pi_{m}\right) \mathrm{c}\left(\pi_{n}\right)+\mathrm{c}\left(\pi_{p}\right)}{\mathrm{s}\left(\pi_{m}\right)^{2}} a+\frac{\mathrm{c}\left(\pi_{m}\right) \mathrm{c}\left(\pi_{p}\right)+\mathrm{c}\left(\pi_{n}\right)}{\mathrm{s}\left(\pi_{m}\right)^{2}} b+c \\
& =\delta a+\varepsilon b+c,
\end{aligned}
$$


while

$$
\begin{aligned}
x_{0} & =-\frac{\mathrm{c}\left(j \pi_{n}\right) \mathrm{c}\left(h \pi_{m}\right)+\mathrm{c}\left(i \pi_{p}\right)}{\mathrm{s}\left(h \pi_{m}\right)^{2}} a-\frac{\mathrm{c}\left(i \pi_{p}\right) \mathrm{c}\left(h \pi_{m}\right)+\mathrm{c}\left(j \pi_{n}\right)}{\mathrm{s}\left(h \pi_{m}\right)^{2}} b^{\prime} \\
& =-\lambda^{\prime} a-\mu^{\prime} b^{\prime}
\end{aligned}
$$

and

$$
\begin{aligned}
x_{0}^{\prime} & =-\frac{\mathrm{c}\left(i \pi_{p}\right) \mathrm{c}\left(h \pi_{m}\right)+\mathrm{c}\left(j \pi_{n}\right)}{\mathrm{s}\left(h \pi_{m}\right)^{2}} a^{\prime}-\frac{\mathrm{c}\left(j \pi_{n}\right) \mathrm{c}\left(h \pi_{m}\right)+\mathrm{c}\left(i \pi_{p}\right)}{\mathrm{s}\left(h \pi_{m}\right)^{2}} b \\
& =-\mu^{\prime} a^{\prime}-\lambda^{\prime} b .
\end{aligned}
$$

The formulas (1) and (2) yield $x_{0}=\lambda a+\mu b$ (in Case (A)) and $x_{0}^{\prime}=\mu a+\lambda b$ (in Case (B)) for certain scalars $\lambda$ and $\mu$ that we do not need to evaluate.

Lemma 12. If $\varepsilon=\delta$, then $n=p$.

Proof. This is clear, since $\varepsilon-\delta=\frac{\left(\mathrm{c}\left(\pi_{n}\right)-\mathrm{c}\left(\pi_{p}\right)\right)\left(1-\mathrm{c}\left(\pi_{m}\right)\right)}{\mathrm{s}\left(\pi_{m}\right)^{2}}$.

As $B$ is non-degenerate and $x-x_{0}$ is perpendicular to both $a$ and $b$ we can see that $x-x_{0}$ must be a multiple of $x_{\perp}$. Thus we may write $x=x_{0}+\omega x_{\perp}$ for some real number $\omega$. If $\omega=0$, then $x=x_{0}$ is a linear combination of $a$ and $b$, and so lies in $\Phi \cap V_{\{a, b\}}=\Phi_{\{a, b\}}$. But since this gives $r_{x} \in\left\langle r_{a}, r_{b}\right\rangle$, contradicting the surjectivity of $\phi$, we conclude that $\omega \neq 0$. Now since $x$ is a root it has length 1 , and so $1=x \cdot x=\left(x_{0}+\omega x_{\perp}\right) \cdot\left(x_{0}+\omega x_{\perp}\right)=x_{0} \cdot x_{0}+\omega^{2} x_{\perp} \cdot c$, which gives

$$
\omega^{2}=\frac{1-x_{0} \cdot x_{0}}{x_{\perp} \cdot c}
$$

since $x_{\perp} \cdot c \neq 0$ by the nondegeneracy of $B$. A similar argument can be applied to $x^{\prime}=x_{0}^{\prime}+\omega x_{\perp}$. An inspection of the expressions for $x_{0}$ and $x_{0}^{\prime}$ in terms of $a$ and $b$ shows that $x_{0} \cdot x_{0}=x_{0}^{\prime} \cdot x_{0}^{\prime}$ and so we obtain the same expression for $\omega^{2}$ in each case. Since $x_{0} \cdot a=x \cdot a$ and $x_{0} \cdot b^{\prime}=x \cdot b^{\prime}$, we find that

$$
\begin{aligned}
\omega^{2} & =\frac{1-x_{0} \cdot x_{0}}{x_{\perp} \cdot c} \\
& =\frac{1+x_{0} \cdot\left(\lambda^{\prime} a+\mu^{\prime} b^{\prime}\right)}{x_{\perp} \cdot c} \\
& =\frac{\mathrm{s}\left(\pi_{m}\right)^{2}\left(\mathrm{c}\left(i \pi_{p}\right)^{2}+\mathrm{c}\left(j \pi_{n}\right)^{2}+2 \mathrm{c}\left(h \pi_{m}\right) \mathrm{c}\left(j \pi_{n}\right) \mathrm{c}\left(i \pi_{p}\right)-\mathrm{s}\left(h \pi_{m}\right)^{2}\right)}{\mathrm{s}\left(h \pi_{m}\right)^{2}\left(\mathrm{c}\left(\pi_{p}\right)^{2}+\mathrm{c}\left(\pi_{n}\right)^{2}+2 \mathrm{c}\left(\pi_{m}\right) \mathrm{c}\left(\pi_{n}\right) \mathrm{c}\left(\pi_{p}\right)-\mathrm{s}\left(\pi_{m}\right)^{2}\right)} \\
& =\frac{\mathrm{s}\left(\pi_{m}\right)^{2} N}{\mathrm{~s}\left(h \pi_{m}\right)^{2} D}
\end{aligned}
$$

where we have $N=-1+\mathrm{c}\left(h \pi_{m}\right)^{2}+\mathrm{c}\left(i \pi_{p}\right)^{2}+\mathrm{c}\left(j \pi_{n}\right)^{2}+2 \mathrm{c}\left(h \pi_{m}\right) \mathrm{c}\left(j \pi_{n}\right) \mathrm{c}\left(i \pi_{p}\right)$, and $D=-1+\mathrm{c}\left(\pi_{m}\right)^{2}+\mathrm{c}\left(\pi_{p}\right)^{2}+\mathrm{c}\left(\pi_{n}\right)^{2}+2 \mathrm{c}\left(\pi_{m}\right) \mathrm{c}\left(\pi_{n}\right) \mathrm{c}\left(\pi_{p}\right)$. It can be checked that $-D$ is the discriminant of $B$ (relative to the basis $\{a, b, c\}$ ), which is negative, as we have noted. Thus $D>0$, and hence $N>0$ (since $\omega \neq 0)$.

Lemma 13. With $m, n, p, i, j$ and $h$ as above, we have $\omega^{2} \leq 1$, with equality if and only if the following conditions all hold: $h=1$ or $m-1 ; j=1$ or $n-1 ; i=1$ or $p-1 ; \mathrm{c}\left(h \pi_{m}\right) \mathrm{c}\left(j \pi_{n}\right) \mathrm{c}\left(i \pi_{p}\right)=\mathrm{c}\left(\pi_{m}\right) \mathrm{c}\left(\pi_{n}\right) \mathrm{c}\left(\pi_{p}\right)$. 
Proof. Since $1 \leq h \leq m-1$ we see that $\left|\mathrm{c}\left(h \pi_{m}\right)\right| \leq \mathrm{c}\left(\pi_{m}\right)$, equality holding if and only if $h=1$ or $m-1$. Similar statements hold for $j$ and $i$, and since

$$
\begin{aligned}
D-N=\left(\mathrm{c}\left(\pi_{m}\right)^{2}-\mathrm{c}\left(h \pi_{m}\right)^{2}\right) & +\left(\mathrm{c}\left(\pi_{n}\right)^{2}-\mathrm{c}\left(j \pi_{n}\right)^{2}\right)+\left(\mathrm{c}\left(\pi_{p}\right)^{2}-\mathrm{c}\left(i \pi_{p}\right)^{2}\right) \\
& +2\left(\mathrm{c}\left(\pi_{m}\right) \mathrm{c}\left(\pi_{n}\right) \mathrm{c}\left(\pi_{p}\right)-\mathrm{c}\left(h \pi_{m}\right) \mathrm{c}\left(j \pi_{n}\right) \mathrm{c}\left(i \pi_{p}\right)\right),
\end{aligned}
$$

we conclude that $D \geq N$, with equality if and only if the four conditions in the statement of the lemma hold. Furthermore, $\mathrm{s}\left(\pi_{m}\right)^{2} \leq \mathrm{s}\left(h \pi_{m}\right)^{2}$, with equality if and only if $h=1$ or $m-1$. Since $\omega^{2}=\mathrm{s}\left(\pi_{m}\right)^{2} N / \mathrm{s}\left(h \pi_{m}\right)^{2} D$, the result follows.

Recall that, in Case (A), $\phi\left(r_{c}\right)=r_{x}$ for some root $x \in \Phi^{+}$, and

$$
x=x_{0}+\omega x_{\perp}=(\lambda+\omega \delta) a+(\mu+\omega \varepsilon) b+\omega c .
$$

In Case (B) we have, similarly,

$$
x^{\prime}=x_{0}^{\prime}+\omega x_{\perp}=(\mu+\omega \delta) a+(\lambda+\omega \varepsilon) b+\omega c .
$$

In either case, it follows from Lemma 3 and Lemma 13 that $\omega=1$, and the four conditions in the statement of Lemma 13 must hold.

If we are in Case (A), then $h=1$ gives $b^{\prime}=b$, while $h=m-1$ gives $b^{\prime}=r_{a} b$; if we are in Case (B), then $h=1$ gives $a^{\prime}=a$, while $h=m-1$ gives $a^{\prime}=r_{b} a$. So we have two possibilities in Case (A) and two in Case (B), as follows:

(A1) $\phi\left(r_{a}\right)=r_{a}, \phi\left(r_{b}\right)=r_{b}$ and $\phi\left(r_{c}\right)=r_{x}$, where $x \in \Phi^{+}$satisfies $x \cdot a=\eta c \cdot a$ and $x \cdot b=\eta c \cdot b$, where $\eta= \pm 1$;

(A2) $\phi\left(r_{a}\right)=r_{a}, \phi\left(r_{b}\right)=r_{a} r_{b} r_{a}$ and $\phi\left(r_{c}\right)=r_{x}$, where $x \in \Phi^{+}$satisfies $x \cdot a=\eta c \cdot a$ and $x \cdot b=-\eta c \cdot b$, where $\eta= \pm 1$;

(B1) $\phi\left(r_{a}\right)=r_{b}, \phi\left(r_{b}\right)=r_{a}$ and $\phi\left(r_{c}\right)=r_{x^{\prime}}$, where $x^{\prime} \in \Phi^{+}$satisfies $x^{\prime} \cdot b=\eta c \cdot a$ and $x \cdot a=\eta c \cdot b$, where $\eta= \pm 1$;

(B2) $\phi\left(r_{a}\right)=r_{b}, \phi\left(r_{b}\right)=r_{b} r_{a} r_{b}$ and $\phi\left(r_{c}\right)=r_{x^{\prime}}$, where $x^{\prime} \in \Phi^{+}$satisfies $x^{\prime} \cdot b=\eta c \cdot a$ and $x^{\prime} \cdot a=-\eta c \cdot b$, where $\eta= \pm 1$.

Replacing $\phi$ by itself followed by conjugation by $r_{a}$ converts Case (A2) into (A1), and similarly replacing $\phi$ by itself followed by conjugation by $r_{b}$ converts Case (B2) into (B1).

Since the form is nondegenerate and $a, b$ linearly independent, the following result is clear.

Lemma 14. Let $\alpha$ and $\beta$ be any scalars. There are at most two vectors $v \in V$ such that $v \cdot a=\alpha, v \cdot b=\beta$ and $v \cdot v=1$; furthermore, if $v \in V$ satisfies these equations, then so does $\sigma v$, where $\sigma: V \rightarrow V$ is the reflection in the plane $V_{\{a, b\}}$.

Recall that Case (B) arises only if $m$ is even and $n>p$. So assume first of all that $m$ is odd. Obviously $x=\eta c$ satisfies $x \cdot a=\eta c \cdot a$ and $x \cdot b=\eta c \cdot b$. By Lemma14 it follows that the only values of $x$ that can satisfy the requirements of Case (A1) above are $x=\eta c$ and $x=\sigma(\eta c)$, where $\eta= \pm 1$. Clearly $x=c$ corresponds to the identity automorphism, while $x=-c$ is not a solution since $-c \notin \Phi^{+}$.

Let $w_{0}=\left(r_{a} r_{b}\right)^{m-1 / 2} r_{a}$, the maximal length element of $\left\langle r_{a}, r_{b}\right\rangle$. Then $\sigma(\eta c)$ is a root if and only if $w_{0}(\sigma(\eta c))$ is a root. Furthermore, since $w_{0}$ fixes $x_{\perp}$, and satisfies $w_{0} a=-b$ and $w_{0} b=-a$, while $\sigma$ fixes $a$ and $b$ and satisfies $\sigma x_{\perp}=-x_{\perp}$, we find that

$$
\begin{aligned}
w_{0}(\sigma c)=w_{0}\left(\sigma\left(\operatorname{proj}(c)+x_{\perp}\right)\right)= & w_{0}\left(\operatorname{proj}(c)-x_{\perp}\right) \\
& =w_{0}(\operatorname{proj}(c))-x_{\perp}=\delta b+\varepsilon a-(c+\delta a+\varepsilon b) .
\end{aligned}
$$


This cannot be a root if $\delta \neq \varepsilon$, since the coefficients of $a$ and $b$ have opposite signs in this case. If $\delta=\varepsilon$, then $\sigma c=w_{0}(-c)$, which is a negative root, while $x=-\sigma c=w_{0} c$ satisfies all the requirements of Case (A1). In this case we see that $\phi$ followed by conjugation by $w_{0}$ interchanges $r_{a}$ and $r_{b}$ and fixes $r_{c}$. Since we have seen in Lemma 12 that $\delta=\varepsilon$ gives $n=p$, this is a graph automorphism of $W$.

Suppose now that $m$ is even. The reflection $\sigma$ appearing in Lemma 14 satisfies $\sigma v=-\left(r_{a} r_{b}\right)^{m / 2} v$ for all $v \in V$, since $\left(r_{a} r_{b}\right)^{m / 2}$ acts as a half-turn on $V_{\{a, b\}}$ and fixes $x_{\perp}$. Thus $\sigma$ preserves the root system $\Phi$. In Case (A) we again see that $x=c$ yields the identity automorphism, and $x=-c$ is inadmissible since $-c \notin \Phi^{+}$. Similarly, $x=\sigma c$ is inadmissible, since $\sigma c=-\left(r_{a} r_{b}\right)^{m / 2} c$ is a negative root. The other possibility is $x=-\sigma c$; in this case it is readily verified that $\phi$ is inner, given by conjugation by $\left(r_{a} r_{b}\right)^{m / 2}$.

Turning finally to Case (B), suppose that $m$ is even and $n>p$. Let $\tau$ be the reflection that interchanges $a$ and $b$ and fixes $x_{\perp}$. By Lemma 14 the only vectors $x^{\prime}$ satisfying $x^{\prime} \cdot b=\eta c \cdot a, x^{\prime} \cdot a=\eta c \cdot b$ and $x^{\prime} \cdot x^{\prime}=1$ are $\tau(\eta c)$ and $\sigma(\tau(\eta c))$. Since $\sigma$ preserves $\Phi$, if we can prove that $\tau c \notin \Phi$, then it will follow that there are no vectors $x^{\prime}$ satisfying the requirements of Case (B1). But $\tau c=c+\alpha(a-b)$ for some scalar $\alpha$, with $\alpha=0$ if and only if $c \cdot(a-b)=0$. This last condition is not satisfied, since $n>p$. Hence the coefficients of $a$ and $b$ in $\tau c$ have opposite signs, showing that $\tau c \notin \Phi$, as required. So Case (B) never arises.

Thus we have shown that in each case $\phi$ is either inner or a product of a graph automorphism and an inner automorphism.

Theorem 15. If $W$ is an infinite rank 3 Coxeter group with $B$ non-degenerate and no infinite bonds, then the automorphism group of $W$ is generated by the inner and graph automorphisms.

\section{The Degenerate CASE}

Now suppose that $W$ is a rank 3 Coxeter group with $B$ degenerate, so that $W \cong \widetilde{A}_{2}, \widetilde{C}_{2}$ or $\widetilde{G}_{2}$, the triples $(m, n, p)$ being, respectively, $(3,3,3),(4,4,2)$ or $(6,3,2)$. For consistency we shall continue to assume that elements of $\Phi$ are normalized to have length 1 , rather than using the standard root systems in types $\widetilde{C}_{2}$ and $\widetilde{G}_{2}$. If $\phi$ is an arbitrary automorphism of $W$, then we have $\phi\left(r_{a}\right)=r_{a^{\prime}}$, $\phi\left(r_{b}\right)=r_{b^{\prime}}$ and $\phi\left(r_{c}\right)=r_{c^{\prime}}$ for some $a^{\prime}, b^{\prime}, c^{\prime} \in \Phi$.

Since $r_{a^{\prime}} r_{b^{\prime}}$ has the same order as $r_{a} r_{b}$ we must have $a^{\prime} \cdot b^{\prime}=\mathrm{c}\left(i \pi_{m}\right)$ for some $i$ coprime to $m$, and since $m$ is 3,4 or 6 this in fact yields $a^{\prime} \cdot b^{\prime}=\varepsilon a \cdot b$ for some $\varepsilon= \pm 1$. Replacing $a^{\prime}$ by $-a^{\prime}$ if necessary, we may assume that $a^{\prime} \cdot b^{\prime}=a \cdot b$. Modifying $\phi$ by a graph automorphism if necessary, we may assume that the parabolic subgroup $\left\langle r_{a^{\prime}}, r_{b^{\prime}}\right\rangle$ is in the same $W$-conjugacy class as $\left\langle r_{a}, r_{b}\right\rangle$, and then modifying $\phi$ by an inner automorphism if necessary, we may assume that $\left\langle r_{a^{\prime}}, r_{b^{\prime}}\right\rangle=\left\langle r_{a}, r_{b}\right\rangle$. Now $\left\{a^{\prime}, b^{\prime}\right\}$ and $\{a, b\}$ are two simple systems in $\Phi_{\{a, b\}}$, and hence lie in the same $\left\langle r_{a}, r_{b}\right\rangle$-orbit. So modifying $\phi$ by a further inner automorphism we may assume that $\left\{r_{a^{\prime}}, r_{b^{\prime}}\right\}=\left\{r_{a}, r_{b}\right\}$.

In type $\widetilde{A}_{2}$ conjugating by the element $r_{a} r_{b} r_{a}$ interchanges $r_{a}$ and $r_{b}$; so in this case we may assume that $a^{\prime}=a$ and $b^{\prime}=b$. In the other two cases $r_{a}$ and $r_{b}$ are not conjugate in $W$, since there are homomorphisms $W \rightarrow\{1,-1\}$ with $a \mapsto-1$ and $b, c \mapsto 1$; furthermore, the conjugacy classes containing $r_{a}$ and $r_{b}$ are distinguished by group-theoretic criteria: in type $\widetilde{C}_{2}$ the reflections conjugate to $r_{a}$ lie in maximal finite subgroups of order 4 , whereas those conjugate to $r_{b}$ do not, and in type $\widetilde{G}_{2}$ 
the reflections conjugate to $r_{b}$ lie in maximal finite subgroups of order 6 , whereas those conjugate to $r_{a}$ do not. So in either case $\phi\left(r_{a}\right)=r_{b}$ is not possible, and hence we may assume that $a^{\prime}=a$ and $b^{\prime}=b$.

Since $r_{b} r_{c^{\prime}}$ has the same order as $r_{b} r_{c}$, it follows (by the same reasoning used above) that $b \cdot c^{\prime}= \pm b \cdot c$, and replacing $c^{\prime}$ by $-c^{\prime}$ if necessary, we may assume that $b \cdot c^{\prime}=b \cdot c$. In type $\widetilde{A}_{2}$ we similarly deduce that $a \cdot c^{\prime}=\varepsilon a \cdot c$ for some $\varepsilon= \pm 1$; however, since $B$ is degenerate the matrix

$$
\left(\begin{array}{ccc}
a \cdot a & a \cdot b & a \cdot c^{\prime} \\
b \cdot a & b \cdot b & b \cdot c^{\prime} \\
c^{\prime} \cdot a & c^{\prime} \cdot b & c^{\prime} \cdot c^{\prime}
\end{array}\right)=\left(\begin{array}{rrr}
1 & -\frac{1}{2} & -\frac{\varepsilon}{2} \\
-\frac{1}{2} & 1 & -\frac{1}{2} \\
-\frac{\varepsilon}{2} & -\frac{1}{2} & 1
\end{array}\right)
$$

must be singular, and so it follows that $\varepsilon=1$. In the other two cases we have $a \cdot c^{\prime}=0$, since $r_{a}$ and $r_{c^{\prime}}$ commute; so we conclude that $a \cdot c^{\prime}=a \cdot c$ in all cases.

We conclude from the above discussion that $c^{\prime}-c$ is in the one-dimensional space which is the orthogonal complement of $V_{\{a, b\}}$. Thus $c^{\prime}=c+\alpha u$ for some scalar $\alpha$, where the vector $u$ is given by

$$
u= \begin{cases}a+b+c & \text { in type } \widetilde{A}_{2}, \\ a+\sqrt{2} b+c & \text { in type } \widetilde{C}_{2}, \\ \sqrt{3} a+2 b+c & \text { in type } \widetilde{G}_{2} .\end{cases}
$$

If the scalar $\alpha$ above is zero, then of course $\phi$ is the identity automorphism. There is only one other possibility for $\alpha$.

Lemma 16. In the above notation, if $\alpha \neq 0$, then $\alpha=-2$.

Proof. Since $\left\{r_{a}, r_{b}, r_{c^{\prime}}\right\}$ generates $W$, it follows that $\left\{a, b, c^{\prime}\right\}$ spans $V$. So $1+\alpha$, the coefficient of $c$ in $c^{\prime}$, is nonzero. Thus, since $c^{\prime} \in \Phi$, it follows from Lemma 3 that $|1+\alpha| \geq 1$. Now let $f: V \rightarrow V$ be the linear transformation defined by $a \mapsto a, b \mapsto b$ and $c \mapsto c^{\prime}$. Then $f$ preserves the bilinear form $B$, and so $f r_{c} f^{-1}=r_{f c}=r_{c^{\prime}}=\phi\left(r_{c}\right)$, and similarly $f r_{a} f^{-1}=\phi\left(r_{a}\right)$ and $f r_{b} f^{-1}=\phi\left(r_{b}\right)$. An obvious induction on length yields $f w f^{-1}=\phi(w)$ for all $w \in W$, and so for all $v \in \Phi$ the element $r_{f v}=f r_{v} f^{-1}=\phi\left(r_{v}\right)$ is a reflection in $W$. Moreover, since $\phi$ is bijective, every reflection in $W$ is obtained in this fashion, and it follows that $v \mapsto f v$ maps $\Phi$ to $\Phi$ bijectively.

The above discussion shows that there is some $v \in \Phi$ such that $f v=c$. Writing $v=\lambda a+\mu b+\nu c$ gives $c=\lambda a+\mu b+\nu(c+\alpha u)$, and equating the coefficients of $c$ gives $\nu(1+\alpha)=1$. But $|\nu| \geq 1$ by Lemma 3, and since we established above that $|1+\alpha| \geq 1$, this shows that $\nu=1+\alpha= \pm 1$. If $\alpha \neq 0$, this gives $\alpha=-2$, as claimed.

It remains to observe that when $\alpha=-2$ the automorphism $\phi$ is the composite of an inner automorphism and a graph automorphism. In type $\widetilde{A}_{2}$ we find that if $w=r_{a} r_{b} r_{a}$, then $w a=-b, w b=-a$ and $w c=c+2 a+2 b=-(c-2 u)$. Thus conjugation by $w$ followed by the graph automorphism that interchanges $r_{a}$ and $r_{b}$ coincides with $\phi$. In the other two cases it is equally easy to check that $\phi$ is given by conjugation by the longest element of $\left\langle r_{a}, r_{b}\right\rangle$. We have thus established that for all three of these groups the full automorphism group is generated by the graph automorphisms and the inner automorphisms. Combined with Theorem [15, this immediately yields Theorem [1. 


\section{REFERENCES}

[1] Brigitte Brink, The dominance minimal roots, J. Algebra 206 (1998), 371-412. MR 99k:20083

[2] N. Bourbaki, Groupes et algèbres de Lie, Chap. 4, 5 et 6, Hermann, Paris, 1968. MR 39:1590

[3] Roger W. Carter, Finite Groups of Lie Type: Conjugacy Classes and Complex Characters, J. Wiley \& Sons, 1985. MR 87d:20060

[4] V. V. Deodhar, On the root system of a Coxeter group, Comm. Algebra 10 (1982), 611-630. MR 83j:20052a

[5] James E. Humphreys, Reflection Groups and Coxeter Groups, Cambridge University Press, 1990. MR 92h:20002

Australian Catholic University, 25A Barker Rd, Strathfield, New South Wales 2135, Australia

E-mail address: b.franzsen@mary.acu.edu.au

School of Mathematics and Statistics, University of Sydney, New South Wales 2006, Australia

E-mail address: R.Howlett@maths.usyd.edu.au 